\title{
Diagnostic performance of apparent diffusion coefficient values for the differentiation of intrahepatic cholangiocarcinoma from gastrointestinal adenocarcinoma liver metastases
}

\author{
Temel Fatih Yilmaz¹, Mehmet Ali Gultekin¹, Hacı Mehmet Turk², Mehmet Besiroglu², \\ Dilek Hacer Cesme ${ }^{1}$, Melih Simsek ${ }^{2}$, Alpay Alkan ${ }^{1}$, Huseyin Toprak ${ }^{1}$
}

\author{
1 Departments of Radiology, Faculty of Medicine, Bezmialem Vakif University, Istanbul, Turkey \\ ${ }^{2}$ Departments of Medical Oncology, Faculty of Medicine, Bezmialem Vakif University, Istanbul, Turkey
}

Radiol Oncol 2022; 56(1): 54-59.

Received 29 July 2021

Accepted 4 November 2021

Correspondence to: Asist. Prof. Temel Fatih Yilmaz, M.D., Department of Radiology, Faculty of Medicine, Bezmialem Vakif University, Istanbul, Turkey. Phone: 90212 4531700; Fax: 90212 6217580; E-mail: temelfatihyilmaz@gmail.com

Disclosure: No potential conflicts of interest were disclosed.

This is an open access article under the CC BY-NC-ND license (http://creativecommons.org/licenses/by-nc-nd/4.0/).

Background. We aimed to investigate whether there is a difference between intrahepatic cholangiocarcinoma (IHCC) and liver metastases of gastrointestinal system (GIS) adenocarcinoma in terms of apparent diffusion coefficient (ADC) values.

Patients and methods. From January 2018 to January 2020, we retrospectively examined 64 consecutive patients with liver metastases due to gastrointestinal system adenocarcinomas and 13 consecutive IHCC in our hospital's medical records. After exclusions, fifty-three patients with 53 liver metastases and 10 IHCC were included in our study. We divided the patients into two groups as IHCC and liver metastases of GIS adenocarcinoma. For mean apparent diffusion coefficient ( $A D C_{\text {mean }}$ ) values, the region of interests (ROI) was placed in solid portions of the lesions. $A D C_{\text {mean }}$ values of groups were compared.

Results. The mean age of IHCC group was $62.50 \pm 13.49$ and mean age of metastases group was 61.15 \pm 9.18 . $A D C_{\text {mean }}$ values were significantly higher in the IHCC group compared to the metastatic group $(p<0.001)$. ROC curves method showed high diagnostic accuracy (AUC $=0.879$ ) with cut-off value of $<1178 \times 10^{-6} \mathrm{~mm}^{2} / \mathrm{s}$ for $A D C_{\text {mean }}$ (Sensitivity $=90.57$, Specificity $=70.0$, positive predictive value $[\mathrm{PPV}]=94.1$, negative predictive value $[\mathrm{NPV}]=58.3$ ) in differentiating adenocarcinoma metastases from IHCC.

Conclusions. The present study results suggest that ADC values have a potential role for differentiation between IHCC and GIS adenocarcinoma liver metastases which may be valuable for patient management.

Key words: cholangiocarcinoma, gatrointestinal system; liver metastases; apparent diffusion coefficient; diffusion weighted image; MRI

\section{Introduction}

Intrahepatic cholangiocarcinoma (IHCC) is the second most common primary malignant lesion of the liver after hepatocellular cancer and estimated 15\% of primary liver cancer worldwide. The incidence of IHCC has been increasing recently. ${ }^{1}$ According to macroscopic appearance, cholangiocarcinoma (CC) is divided into three types: mass forming type, periductal infiltrative type and intraductal grow- 
ing type. ${ }^{1-2}$ Mass forming type CC is the most common type with a rate of $60 \% .^{3}$ Diffusion weighted image (DWI) usually is added to standard abdomen protocols, because it is a rapid technique and talented of detecting most liver masses in patients with supposed malignant disease. It may be difficult to differentiate between IHCC and gastrointestinal system (GIS)-derived adenocarcinoma even when contrast is given, and DWI can be helpful in differential diagnosis in these cases. ${ }^{4}$

DWI provides diagnostic value in differentiation of benign and malignant liver masses. It gives information about cellularity of tissues and integrity of cell membranes. DWI increases the sensitivity of detection for liver metastases when combined with dynamic contrast enhanced upper abdomen MRI. DWI has been used in characterization of metastatic and primary liver tumors. ${ }^{5}$ The sensitivity of using DWI in addition to routine imaging in detecting malignancy was reported as $94.9 \%$ and the specificity as $97.8 \%{ }^{6}$ To avoid unnecessary diagnostic and therapeutic interventions, differentiation between IHCC and metastases of adenocarcinomas is very significant, because they have different treatment options and prognosis. It would be valuable to precisely differentiate the liver metastases of GIS from IHCC based on apparent diffusion coefficient (ADC) values. Because it's sometimes hard to differentiate even based on the histological analysis since all these tumors are adenocarcinomas. We aimed to investigate whether there is a difference between IHCC's and adenocarcinoma liver metastases from GIS origin in terms of ADC values.

The goal of this study was to evaluate the value of DWI, using the mean ADC value for distinguishing IHCC from liver metastases of adenocarcinomas originating in the gastrointestinal tract.

\section{Patients and methods}

\section{Patients}

We consecutively reviewed medical records of patients who had a diagnosis or an imaging study showing IHCC or metastasis between January 2018 to January 2020. Inclusion criteria were determined as histopathological confirmation of the primary or metastatic lesion GIS adenocarcinoma arising from stomach, colon, and pancreas with liver metastases at the initial diagnosis, and CC with no history of chemotherapy at initial imaging and the presence of pretreatment MRI of the abdomen with a proper DWI.

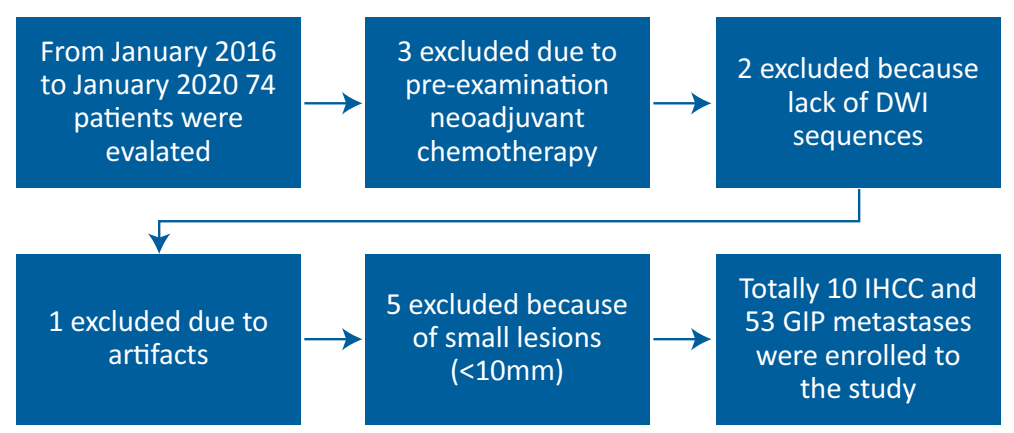

FIGURE 1. Flow-chart of the study showing the exclusion criteria of the patients.

Exclusion criteria were determined as pre-examination neoadjuvant chemotherapy for the primary tumor $(n=3)$, lack of DWI sequence $(n=2)$, heavy image artefacts and technical reasons $(n=1)$, and lesions smaller than $10 \mathrm{~mm}$ with difficult to measure ADC values $(n=5)$. We only included one major metastatic lesion from each patient to provide study homogeneity. Finally, a total of 53 patients with 53 liver metastases and 10 patients with IHCC were included in our study (Figure 1). We divided the patients into two groups as IHCC's ( $n=10$ patients) and liver metastases of GIS adenocarcinoma ( $n=53$ patients with 53 lesions). In our study group, there were no underlying chronic liver diseases and no metastases contained mucinous components in it.

The study protocol was approved by our institution's ethics committee. All procedures performed in the studies involving human participants were in accordance with the ethical standards of the institutional and/or national research committee and with the 1964 Helsinki Declaration and its later amendments or comparable ethical standards. Informed consent was obtained from all individual participants included in the study.

\section{Histopathologic analysis}

Diagnosis of all IHCCs and primary GIS adenocarcinomas were confirmed histopathologically. For IHCC's, primary tumor sites were right hepatic lobe $(n=4)$, and left hepatic lobe $(n=6)$, Histopathological diagnosis was obtained by percutaneous tru-cut biopsy.

For the metastasis group, primary tumor sites were colorectal $(n=32)$, gastric $(n=9)$, and pancreas $(n=12)$. Tissue samples of colorectal and gastric adenocarcinomas were obtained through endoscopic biopsy samples. For pancreatic adenocarcinomas, endosonographic-guided fine needle 


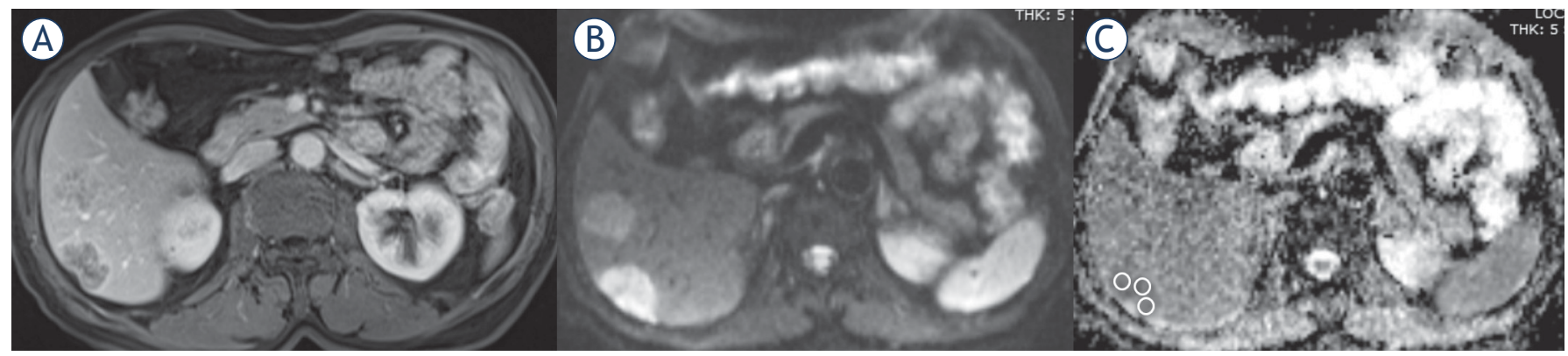

FIGURE 2. 59-year-old male with liver metastases due to colorectal adenocarcinoma. Contrast-enhanced axial Tl-weighted (TIW) (A), diffusion weighted image (DWI) obtained at b value of $800 \mathrm{~s} / \mathrm{mm}^{2}$ (B) and apparent diffusion coefficient (ADC) maps (C) with free hand ROI placement technique.

aspiration biopsy $(\mathrm{n}=7)$, percutaneous tru-cut biopsy of metastatic liver lesions $(\mathrm{n}=3)$, and surgical biopsy $(n=2)$ were done.

\section{MRI protocol}

All patients underwent MRI using a 1.5-T system (Siemens, Avanto, Erlangen, Germany). T1-W inand out-of-phase (TR/TE, 128/4.90 and 128/2.37; NEX, 1; the FOV of 38 to $50 \mathrm{~cm}$; $5 \mathrm{~mm}$ thickness and $2 \mathrm{~mm}$ intersection gap), T2-weighted axial (TR/TE, 2000/120; NEX, 1; the FOV of 38 to $50 \mathrm{~cm}$; $5 \mathrm{~mm}$ thickness and $2 \mathrm{~mm}$ intersection gap), fast spin echo T2-W axial (TR/TE, 2000/117; NEX, 1; the FOV of 38 to $50 \mathrm{~cm} ; 5 \mathrm{~mm}$ section thickness and $2 \mathrm{~mm}$ intersection gap) and T2-W coronal images were performed (TR/TE, 1400/106; NEX, 1; the FOV of 38 to $50 \mathrm{~cm} ; 5 \mathrm{~mm}$ section thickness and $1 \mathrm{~mm}$ intersection gap). The DWI images were obtained at b-values of 50, 400, and $800 \mathrm{~s} / \mathrm{mm}^{2}$ (TR/TE, 7300/78; NEX, 2; FOV 38-50 cm; slice thickness $5 \mathrm{~mm}$ and no intersection gap). Pre and postcontrast fat-saturated T1-W axial (VIBE) (TR/TE, 4.90/2.39; NEX, 1; and the FOV of 38 to $50 \mathrm{~cm} ; 3 \mathrm{~mm}$ section thickness and no intersection gap) were performed. Dynamic imaging was performed after a rapid bolus of gadolinium-diethylenetriamine pentaacetic acid, 0.1 $\mathrm{mmol} / \mathrm{kg}$ body weight, intravenously at a rate of $1.5 \mathrm{~mL} / \mathrm{s}$, followed by a $30 \mathrm{~mL}$ saline flush using a power injector. Contrast-enhanced dynamic images were performed in arterial phase, portal venous phase, and interstitial phase in the axial plane and in interstitial phase in the coronal plane.

\section{Image analysis}

Image analysis and region of interests (ROI) placement was made by two abdominal radiologists who had ten and eleven years' experience of abdominal radiology. The morphological features were evaluated as follows: 1- lesion size, measurements were made in the axial plane on contrast enhanced T1weighted (T1W) images at the largest diameter. 2-lesion localization (right or left lobe of the liver). 3-ADC values of the metastases and IHCC's. For $\mathrm{ADC}_{\text {mean }}$ values, the ROIs were placed over the three different enhancing solid portions of the lesions on contrast enhanced T1W images blinded to ADC maps. Conventional T2-weighted (T2W) and contrast enhanced T1W images were used as reference to determine the enhanced portions of the lesion areas and to avoid the cystic or necrotic parts of the lesions. Final $\mathrm{ADC}_{\text {mean }}$ values were calculated as the average of the ADC values obtained from 3 different ROIs (Figure 2-3). We used synapse 3D ${ }^{\circledR}$ (Fujifilm Medical, Tokyo, Japan) and Leonardo console (software version 2.0, Siemens) to evaluate metastatic liver lesions and to calculate ADC values of metastatic liver lesions.

\section{Statistical analysis}

Statistical analysis was performed using the IBM SPSS Statistics 26.0 statistical software (Armonk, NY: IBM Corp.). The Kolmogorov-Smirnov test was used for normality. non-parametric MannWhitney-U test was used in the comparison of the $\mathrm{ADC}_{\text {mean }}$ values of the IHCC and adenocarcinoma groups from the enhanced solid metastases and the lesion sizes. Descriptive statistics are presented as median $(50 \%)$ and interquartile range $(\mathrm{IQR}=\mathrm{Q} 3(75 \%)-\mathrm{Q} 1(25 \%))$ values. $\mathrm{p}$ value below 0.05 was accepted as statistically significant. ROC curves were evaluated to determine the cut-off value to differentiate between $\mathrm{ADC}_{\text {mean }}$ values of IHCC and adenocarcinoma metastases. 


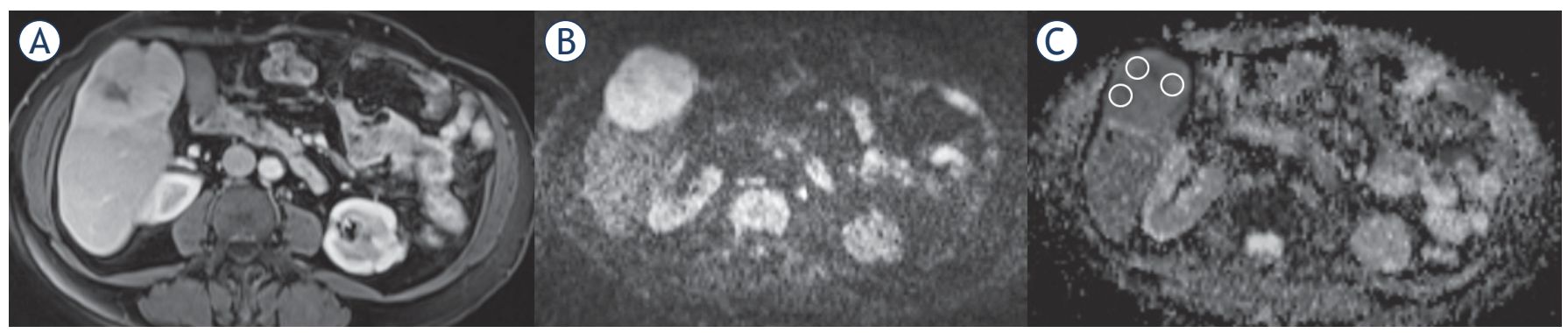

FIGURE 3. 58-year-old female with an expansile liver mass with central hypovascular fibrous stroma and peripheral contrast enhancement on contrastenhanced axial Tl-weighted (TIW) (A) images. diffusion weighted image (DWI) obtained at b value of $800 \mathrm{~s} / \mathrm{mm}^{2}$ (B) and apparent diffusion coefficient $(A D C)$ maps $(C)$ with region of interests (ROI) placement with three different contrast enhancing area for calculating $A D C_{\text {mean }} v{ }^{2}$ lues.

\section{Results}

IHCC was diagnosed in 10 patients with 10 lesions and adenocarcinoma was diagnosed in 53 patients with 53 liver metastases. There was no statistically significant difference between IHCC and adenocarcinoma in terms of age. Demographic features were summarized at Table 1.

$\mathrm{ADC}_{\text {mean }}$ values were significantly higher in the IHCC group compared to the adenocarcinoma group $(\mathrm{p}<0.001)$ (Figure 4$)$. These results are summarized in Table 2.

ROC curves method showed diagnostic accuracy for $\mathrm{ADC}_{\text {mean }}$ ( $\left.\mathrm{AUC}=0.879\right)$. Cut-off value was $<$ $1178 \times 10^{-6} \mathrm{~mm}^{2} / \mathrm{s}$ for $\mathrm{ADC}_{\text {mean }}$ (Sensitivity $=90.57 \%$, Specificity $=70.0 \%$, positive predictive value $[P P V]=94.1$, negative predictive value $[\mathrm{NPV}]=58.3)$ in differentiating adenocarcinoma metastases from IHCC's with the 95\% confidence interval (Figure 5). The AUC rates showed that $\mathrm{ADC}_{\text {mean }}$ values were statistically significant in differentiating the two groups $(\mathrm{p}<0.0001)$.

\section{Discussion}

Gastrointestinal cancer is from the most common malignant tumors worldwide with rising incidence. ${ }^{7}$ Surgery is now the primary treatment for gastrointestinal cancer. Liver metastasis occurs in approximately $45 \%$ of patients. ${ }^{8}$

IHCC originates from small intrahepatic bile ducts and grows through adjacent liver parenchyma. IHCC typically occurs as a large mass, which is difficult to differentiate from a metastatic focus of adenocarcinoma. The only curative treatment of IHCC is surgery and even with surgery its 5-year survival rates remain at $39-41 \% .{ }^{9}$ In contrast there is no surgical treatment option for some metastatic GIS tumors. ${ }^{10}$

IHCC is hypointense on T1-weighted (T1W), and hyperintense on $\mathrm{T} 2 \mathrm{~W}$ imaging relative to liver parenchyma. The grade of hyperintensity on $\mathrm{T} 2 \mathrm{~W}$ imaging frequently depends on the quantity of fibrosis, necrosis, and mucin within the tumor. ${ }^{11}$ The imaging features of IHCC have been further described, with the targetoid appearance being one of the most common characteristics. ${ }^{12}$

Magnetic resonance (MR) DWI is a technique that provides image contrast by free water mol-

TABLE 1. Univariate analysis of patient characteristics for gastrointestinal system (GIS) liver metastases and intrahepatic cholangiocarcinoma (IHCC)

\begin{tabular}{|c|c|c|c|c|}
\hline $\begin{array}{l}\text { Patient } \\
\text { characteristics }\end{array}$ & $\begin{array}{c}\text { Number } \\
\text { of patients } \\
(n=63)\end{array}$ & $\begin{array}{c}\text { IHCC } \\
(n=10)\end{array}$ & $\begin{array}{c}\text { Liver } \\
\text { metastases } \\
(n=53)\end{array}$ & $P$ value \\
\hline Age (years) & $61.4 \pm 9.93$ & $62.50 \pm 13.49$ & $61.15 \pm 9.18$ & 0.679 \\
\hline Gender & & & & $<0.001$ \\
\hline Male & 35 & 2 & 33 & \\
\hline Female & 28 & 8 & 20 & \\
\hline $\begin{array}{l}\text { Diameter }(\mathrm{mm}) \\
\text { Location }\end{array}$ & $47 \pm 31.27$ & $82.70 \pm 28.58$ & $40.26 \pm 27$ & $\begin{array}{l}<0.001 \\
<0.001\end{array}$ \\
\hline Right lobe & 24 & 2 & 38 & \\
\hline Left lobe & 43 & 8 & 15 & \\
\hline
\end{tabular}

TABLE 2. Apparent diffusion coefficient (ADC) values of intrahepatic cholangiocarcinoma (IHCC) and gastrointestinal system (GIS) liver metastases

\begin{tabular}{lc}
\hline Patient Groups & ADC $\times 10^{-6} \mathrm{~mm} / \mathrm{sn}^{2}$ (median, IQR) \\
IHCC & $(1293.0),(1422.0-951.75)$ \\
GIS metastases & $(861.0),(1053.0-695.0)$ \\
\hline
\end{tabular}

$I Q R=$ interquartile range 


\section{Mean ADC values of liver metastases and IHCC}

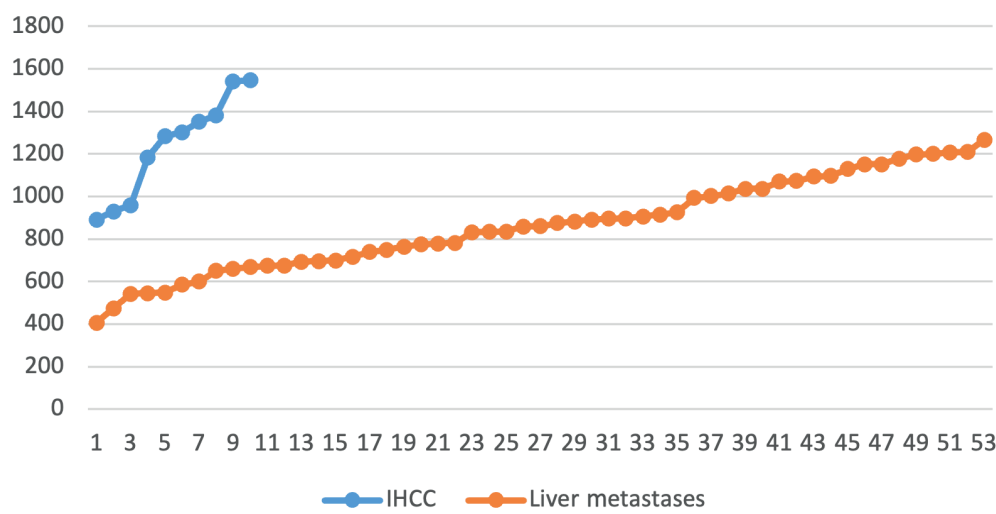

FIGURE 4. Mean apparent diffusion coefficient $\left(A D C_{\text {mean }}\right)$ values of liver metastases of gastrointestinal system adenocarcinomas and intrahepatic cholangiocarcinoma (IHCC). added to routine abdominal MR imaging since it is very sensitive to detect liver malignancies. ADC is a measure of the magnitude of diffusion within tissue and is commonly clinically calculated using DWI. ADC values reflect the structure of masses and vary in different tumor types. Lower ADC values may reflect the hypercellularity of these tumors, and decreased intra and extracellular space.

Mungai et al. reported mean ADC values of CC as $0.970 \times 10^{-3} \mathrm{~mm} / \mathrm{sn}^{2}$ and metastases as $0.947 \mathrm{x}$ $10^{-3} \mathrm{~mm}^{2} / \mathrm{sn}$ and these results were not statistically significant. ${ }^{4}$ This may be a result of evaluation of metastases arising from any origin and the evaluation of all subtypes of CC. In our study we have found median ADC value of IHCC $1293 \times 10^{-6} \mathrm{~mm}^{2} /$ sn and mean ADC value of metastases of GIS 861 $x 10^{-6} \mathrm{~mm}^{2} / \mathrm{sn}$ and this was statistically significant $(\mathrm{p}<0.0001)$. Decreased ADC values in GIS liver metastases may be attributed to hypercellularity. However increased ADC values in IHCC may be the result of decreased cellularity due to fibrotic changes compared to metastases.

Drevelegas et al. demonstrated that mean ADC value in 12 patients with liver CC was $1.34 \pm 0.27$ $\times 10^{-3} \mathrm{~mm}^{2} / \mathrm{s}$ and $1.11 \pm 0.295 \times 10^{-3} \mathrm{~mm}^{2} / \mathrm{s}$ in $51 \mathrm{pa}-$ tients with secondary liver malignancy. ${ }^{13}$ They only concluded that primary liver tumors have higher ADC values than secondary ones. The result of this study supports our outcomes.

Namimoto et al. found the mean ADC value of $1.51 \pm 0.47 \times 10^{-3} \mathrm{~mm}^{2} / \mathrm{s}$ in IHCC and $1.23 \pm 0.32 \times$ $10^{-3} \mathrm{~mm}^{2} / \mathrm{s}$ in metastatic liver lesions. These results are in accordance with the results in our study. ${ }^{6}$

Lee et al. in their study on 91 patients, stated good prognosis and survival of IHCCs when areas with diffusion restriction are dominant. ${ }^{14}$ But they do not provide ADC values of masses in their study. Yamada et al. notified that low ADC value is associated with poor differentiation and prognosis which has rich fibrotic stroma. The researchers, who divided the patients into two groups as high or low ADC, showed that the prognosis was poor in the tumors with decreased ADC. This result conflicts with the results of previous studies by Lee $\mathrm{et} \mathrm{al.} .^{15}$

In our study, we did not make prognostic grouping of our IHCC patients. In our study, we found significantly higher ADC values in IHCC patients compared to GIS adenocarcinoma liver metastases. We distinguished these tumors with a cut off $\mathrm{ADC}_{\text {mean }}$ value of $1178 \times 10-6 \mathrm{~mm}^{2} / \mathrm{s}$ and $90.57 \%$ sensitivity. We speculated that the rich desmoplastic stroma of IHCC is effective in revealing the ADC difference in the differentiation from liver metastases of GIS. 
In a recent study, Kovac et al. investigated the contribution of ADC values in the differentiation between solitary hypovascular liver metastases and IHCC. ${ }^{16}$ They reported lower ADC values in the peripheral enhancing areas and higher in the central parts in IHCC compared to solitary hypovascular metastases. Authors explained that high ADC values in the central part of IHCC are related to rich fibrous tissue. ${ }^{16}$ In our study, ADC values were obtained from enhancing solid area and we found higher ADC values compared to GIS metastases. High ADC values in IHCC could be attributed to desmoplastic stromal changes and decreased cellularity within the tumor.

We thought that the reproducibility of ADC measurement may be limited or needs more effort in clinical practice, but it provides significant diagnostic clues in differential diagnosis of such liver malignancies.

There were several limitations in the current study. First this is a retrospective study and has relatively small sample size, especially for the IHCC group because of the rarity of these tumors. Second, the fibrous intensity and differentiation levels of tumors was not assessed by histopathologically. Furthermore, the manual placement of ROI, as a known limitation in all ROI-based studies, may have led to biased results. In addition, the histopathological confirmation of liver metastases was made from primary tumor localization. But this situation was ignored because of low probability of synchronous IHCC and GIS metastases.

\section{Conclusions}

As far as we know, this is the first study that particularly demonstrates the utility of ADC values in differentiation of IHCC from GIS adenocarcinoma liver metastases. Our study results suggest that ADC values have a potential role for differentiation between IHCC and GIS liver metastases which may be valuable for patient management. Further larger-scale studies are needed to establish the relationship between IHCC and different tumor origins in terms of $\mathrm{ADC}$ values.

\section{References}

1. Shin HR, Oh JK, Masuyer E, Curado MP, Bouvard V, Fang YY, et al. Epidemiology of cholangiocarcinoma: an update focusing on risk factors. Cancer Sci 2010; 101: 579-85. doi: 10.1111/j.1349-7006.2009.01458.x

2. Zhou Y, Zhou G, Gao X, Xu C, Wang X, Xu P. Apparent diffusion coefficient value of mass-forming intrahepatic cholangiocarcinoma: a potential imaging biomarker for prediction of lymph node metastasis. Abdom Radiol 2020; 45: 3109-18. doi: 10.1007/s00261-020-02458-x.

3. Lim JH. Cholangiocarcinoma: morphologic classification according to growth pattern and imaging findings. AJR Am J Roentgenol 2003; 181: 819-27. doi: 10.2214/ajr.181.3.1810819

4. Mungai F, Morone M, Villanacci A, Bondioni MP, Mazzoni LN, Grazioli L, et al. Diffusion weighted MR and apparent diffusion coefficient measurement in classification and characterization of noncystic focal liver lesions: does a clinical role exist? Medicine (Baltimore) 2014; 93: e40. doi: 10.1097/ MD.0000000000000040

5. Shenoy-Bhangle A, Baliyan V, Kordbacheh H, Guimaraes AR, Kambadakone A. Diffusion weighted magnetic resonance imaging of liver: Principles, clinical applications and recent updates. World J Hepatol 2017; 9: 1081-91. doi: 10.4254/wjh.v9.i26.1081

6. Namimoto $T$, Nakagawa $M$, Kizaki $Y$, Itatani R, Kidoh $M$, Utsunomiya D, et al. Characterization of liver tumors by diffusion-weighted imaging: Comparison of diagnostic performance using the mean and minimum apparent diffusion coefficient. J Comput Assist Tomogr 2015; 39: 453-61. doi: 10.1097/ RCT.0000000000000228

7. Rawla P, Barsouk A. Epidemiology of gastric cancer: global trends, risk factors and prevention. Prz Gastroenterol 2019; 14: 26-38. doi: 10.5114/ pg.2018.80001

8. Zheng DX, Ning AY, Levoska MA, Xiang L, Wong C, Scott JF, et al. Predicting liver metastasis of gastrointestinal tract cancer by diffusion-weighted imaging of apparent diffusion coefficient values. World J Gastroenterol 2016; 22: 3031-7. doi: 10.3748/wjg.v22.i10.3031

9. Wang K, Zhang H, Xia Y, Liu J, Shen F. Surgical options for intrahepatic cholangiocarcinoma. Hepatobiliary Surg Nutr 2017; 6: 79-90. doi: 10.21037/ hbsn.2017.01.06

10. Vanderveen KA, Hussain HK. Magnetic resonance imaging of cholangiocarcinoma. Cancer Imaging 2004; 4: 104-15. doi: 10.1102/1470-7330.2004.0018

11. Maetani $Y$, Itoh $K$, Watanabe $C$, Shibata $T$, Ametani $F$, Yamabe $H$, et al. MR imaging of intrahepatic cholangiocarcinoma with pathologic correlation. AJR Am J Roentgenol 2001; 176: 1499-507. doi: 10.2214/ajr.176.6.1761499

12. Kele PG, van der Jagt EJ. Diffusion weighted imaging in the liver. World J Gastroenterol 2010; 16: 1567-76. doi: 10.3748/wjg.v16.i13.1567

13. Drevelegas K, Nikiforaki K, Constantinides M, Papanikolaou N, Papalavrentios L, Stoikou I, et al. Apparent diffusion coefficient quantification in determining the histological diagnosis of malignant liver lesions. J Cancer 2016; 7: 730-5. doi: 10.7150/jca.14197

14. Lee J, Kim SH, Kang TW, Song KD, Choi D, Jang KT. Mass-forming intrahepatic cholangiocarcinoma: diffusion-weighted imaging as a preoperative prognostic marker. Radiology 2016; 281: 119-28. doi: 10.1148/radiol.2016151781

15. Yamada S, Morine Y, Imura S, Ikemoto T, Arakawa Y, Saito Y, et al. Prognostic prediction of apparent diffusion coefficient obtained by diffusion-weighted $\mathrm{MRI}$ in mass-forming intrahepatic cholangiocarcinoma. J Hepatobiliary Pancreat Sci 2020; 27: 388-95. doi: 10.1002/jhbp.732

16. Kovač JD, Galun D, Đurić-Stefanović A, Lilić G, Vasin D, Lazić L, et al. Intrahepatic mass-forming cholangiocarcinoma and solitary hypovascular liver metastases: is the differential diagnosis using diffusion-weighted MRI possible? Acta Radiol 2017; 58: 1417-26. doi: 10.1177/0284185117695666 